\title{
"Liver parenchyma dissecting-first" method facilitates the Glissonean pedicle approach in anatomical laparoscopic hepatolobectomy
}

\author{
Liang Xiao, Zhiming Wang, Ledu Zhou \\ Department of Liver Surgery, Xiangya Hospital, Central South University, Changsha, China \\ Contributions: (I) Conception and design: L Zhou; (II) Administrative support: Z Wang; (III) Provision of study materials or patients: Z Wang, L \\ Zhou; (IV) Collection and assembly of data: L Xiao, L Zhou; (V) Data analysis and interpretation: L Xiao, L Zhou; (VI) Manuscript writing: All \\ authors; (VII) Final approval of manuscript: All authors. \\ Correspondence to: Ledu Zhou, MD. Department of Liver Surgery, Xiangya Hospital, Central South University, Changsha, China. \\ Email: csuzld@yeah.net.
}

\begin{abstract}
Background: Anatomical laparoscopic hepatolobectomy (ALH) is a difficult and challenging operation, and avoiding massive hemorrhage during parenchyma transection remains one of its major concern. Glissonean pedicle approach (GPA) has achieved great success in open liver surgery due to its simplicity and reproducibility, but it is difficult to apply in ALH for the limited vision and motion. We thus aimed to introduce a modified method to facilitate the GPA in ALH.

Methods: In this retrospective study, in 15 patients who underwent ALH (at least 2 couinaud segments), the liver parenchyma was dissected at first (LPDF) adequately under intermittent total inflow control to reveal the Glissonean pedicle (GP) sufficiently for safe and precise transection. The technical details of LPDF for different types of hepatolobectomy and a modified laparoscopic Pringle maneuver are described, and the surgical outcomes are reviewed.

Results: LPDF was applied in all patients, and intraoperative blood transfusion was not needed. LPDF facilitated GPA in ALH without any severe morbidity or mortality.

Conclusions: LPDF is a safe and effective technique, it reduces the difficulties in inflow occlusion in GPA and can promote the application of ALH. Further clinical control trails are needed to validate the superiority of LPDF compared with GPA in ALH.
\end{abstract}

Keywords: Laparoscope; liver; anatomical resection; Glissonean pedicle

Submitted May 13, 2020. Accepted for publication Jul 29, 2020.

doi: $10.21037 /$ atm-20-4674

View this article at: http://dx.doi.org/10.21037/atm-20-4674

\section{Introduction}

With the enrichment of surgeon's experience and the improvement in surgical instruments, anatomical laparoscopic hepatolobectomy (ALH) has been increasingly performed during the last decade. However, extended surgery time and massive intraoperative bleeding are two main factors that contribute to morbidity and mortality in hepatectomy (1-4), and avoiding massive hemorrhage during parenchyma transection remains a major concern for the manipulation restrictions of laparoscopic devices during the operation.

In the past, the extrahepatic artery, portal vein, and bile duct in the Glissonean pedicle (GP) were exposed separately before ligation and transection, and then the liver parenchyma was dissected in liver resection. However, this method is complicated and time-consuming, and it could result in false injury to the vascular and biliary structures in cases of anatomical variation and the surgeons are lack of experience. More recently, the GP approach (GPA) has been widely used as an improved technique for inflow 
occlusion in open liver resection due to its simplicity and reproducibility $(5,6)$. However, GPA remains a challenge in ALH due to the added difficulties of motion and vision. Machado et al. (7) introduced a laparoscopic intrahepatic GP (IGP) approach by making three incisions around the first porta hepatis. The right, right posterior, and anterior GPs could be occluded respectively by introducing a large vascular clamp via a different combination of the three incisions. Then, the clamp is replaced by a laparoscopic vascular stapler, which is fired when the ischemic delineation on the liver surface is identified. However, this method has entails some blindness; it can also be dangerous when there are vessels underneath the incisions and the surgeons are inexperienced, thus increasing the risk of bleeding. Furthermore, using the stapler before parenchyma dissection and the full exposure of the GP may lead to false injury of the remaining Glissonean structures (8).

Herein, we introduce a simplified technique named "liver parenchyma dissecting-first" (LPDF) method for the GPA in $\mathrm{ALH}$, which aims to dissect the liver parenchyma adequately under intermittent total inflow control at first to reveal the GP sufficiently for safe and precise transection, and reduce intraoperative blood loss. We present the following article in accordance with the STROBE reporting checklist (available at http://dx.doi.org/10.21037/atm-20-4674).

\section{Methods}

\section{Patients}

The inclusion criteria were as follows: (I) patient had hepatic tumor (either benign or malignant); (II) patient needed hepatolobectomy (at least two couinaud segments); (III) the liver function was Child-Pugh class A or B. The exclusion criteria was as follows: (I) patient needed only segmental hepatectomy (one couinaud segment); (II) there was tumor thrombus in the main branches of portal vein or hepatic vein; (III) patient had jaundice (total serum bilirubin $>17.1 \mu \mathrm{mol} / \mathrm{L}$ ) or ascites detected by preoperative ultrasound; (IV) patient had any other organ infection or dysfunction.

There were 15 patients ( 10 males and 5 females) underwent ALH using the LPDF method by the same team of surgeons in our department between June 2019 and April 2020 (considering the relative technical simplicity of left lateral lobectomy, it was not included in the current study). The mean age of these patients was 54.6 years (ranging from 33 to 65 years). Twelve patients had chronic hepatitis type B (CHB) related hepatocellular carcinoma (HCC). One patient had metastatic colon carcinoma to the liver, and two patients had giant hemangioma. The liver function was Child-Pugh class A in all patients. The median ECOG PS score was 0 (ranging 0 to 1 ). Surgical outcomes were analyzed retrospectively. These patients provided informed consent for the indication of the surgery, the possible postoperative complications, prognosis, and the timing of conversion to open surgery, and for the publication of their cases and any accompanying images. All procedures performed in this study involving human participants were in accordance with the Declaration of Helsinki (as revised in 2013). The study protocol was approved by the hospital institutional review board.

\section{Surgical technique}

After general anesthesia, a $10-\mathrm{mm}$ incision was made on the top of the navel, through which a Veress needle was placed into the abdominal cavity to deliver $\mathrm{CO}_{2}$ gas. The pneumoperitoneum pressure was maintained between $12-14 \mathrm{mmHg}$ using two insufflators. Usually, the operating surgeon stood on the right side of the patient for both right and left hepatectomy, while the two assistants stood on the left side. The trocars were placed in a location that optimized the operation (Figure 1). Abdominal cavity exploration for possible metastasis and ultrasound liver examination for the location of lesions and hepatic veins were performed routinely. The round ligament, the falciform and coronary ligaments were dissected using an ultrasound scalpel to reveal the second porta hepatis (suprahepatic inferior vena cava, sIVC). The gallbladder was removed when necessary to expose the hepatic hilar better.

Laparoscopic occlusion of the hepatoduodenal ligament was implemented with a urinary catheter (approximately $10 \mathrm{~cm}$ in length), which was then clamped with a hem-olok clip to prevent loosening. For total inflow occlusion to the liver, the catheter was pulled up alternatively using two dissecting forceps for several times and then clamped with another hem-o-lok clip close to the hepatoduodenal ligament to tighten it up. To relieve occlusion, the second hem-o-lok clip (the one close to the hepatoduodenal ligament) was transected by an ultrasound scalpel as shown in Figure 2. Parenchyma dissection was carried out using an ultrasound scalpel under intermittent total inflow control (5-min release between every 15 -min occlusion) and low central venous pressure (CVP) $\left(0-5 \mathrm{cmH}_{2} \mathrm{O}\right)$ anesthesia (9) to reduce hemorrhage both from the GPs and hepatic veins. 


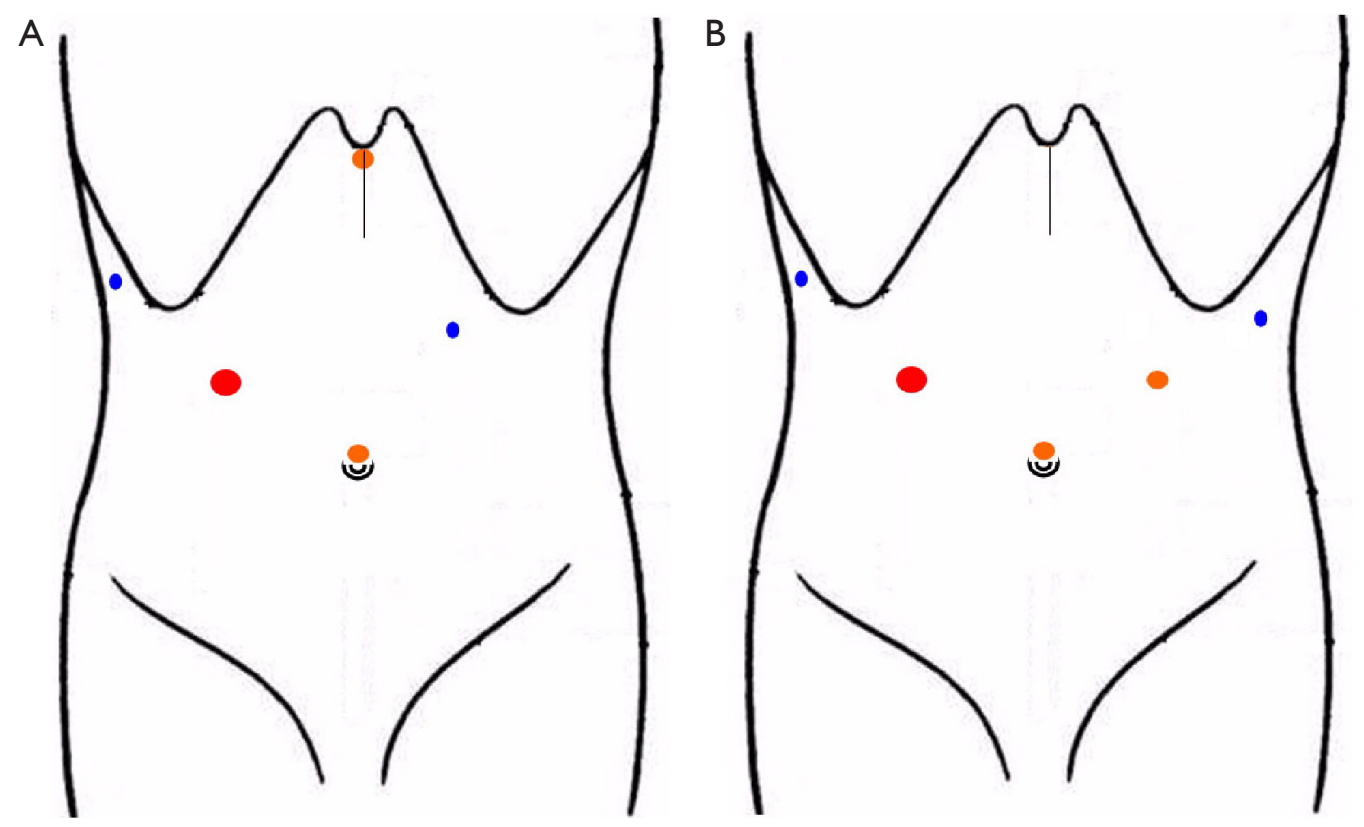

Figure 1 Trocar placement for anatomical laparoscopic hepatectomy. Trocar placement for right (A) and left (B) hepatectomy. Usually, the operating surgeon stands on the right side of the patient while the two assistants stand on the left side. The laparoscope is inserted into the abdominal cavity through a 10-mm trocar (orange) placed on the top of the navel. Then, one 12-mm trocar (red), two 5-mm trocars (blue), and one more $10-\mathrm{mm}$ trocar (orange) are placed in a manner that optimizes manipulation. The removed specimen is put into a sterile canvas bag and taken out through a longitudinal subxiphoid incision (about 6-10 cm in length).
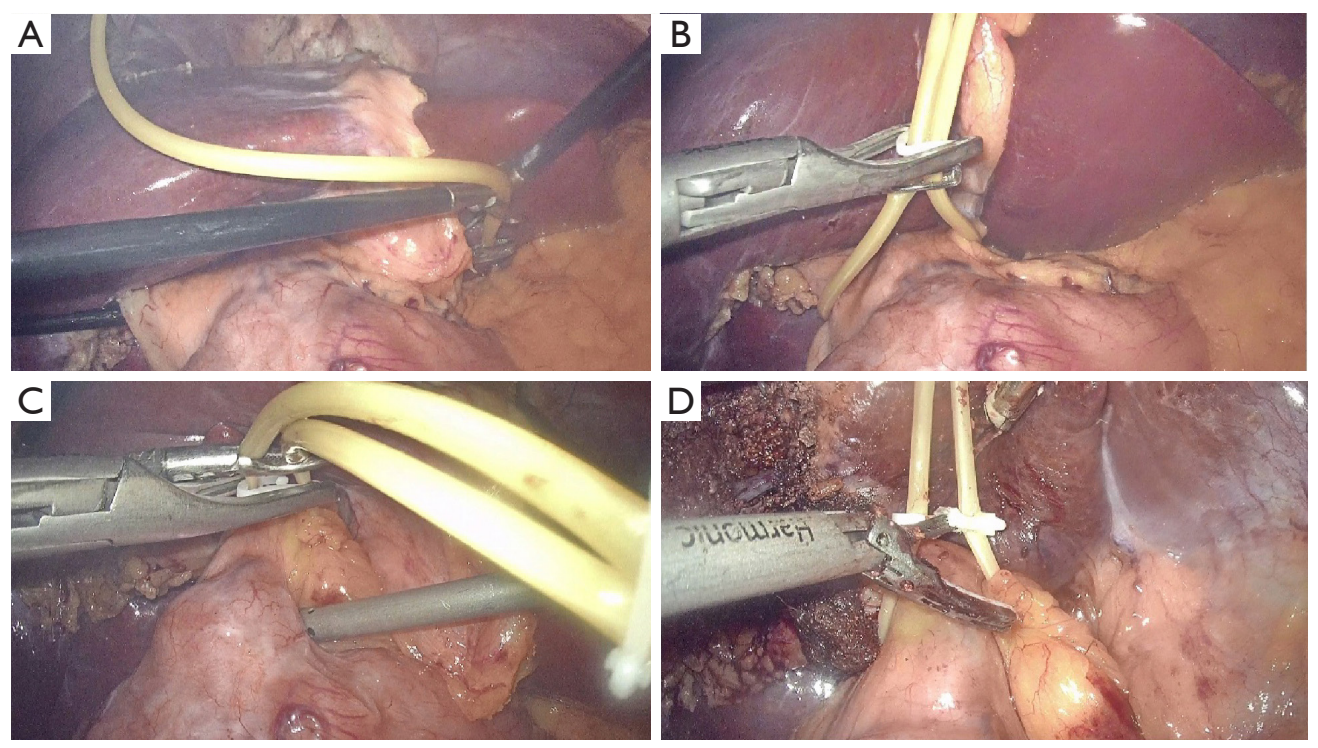

Figure 2 A modified method for laparoscopic Pringle maneuver. Laparoscopic encircling of the hepatoduodenal ligament is carried out using a urinary catheter (approximately $10 \mathrm{~cm}$ in length, A), which is then clamped with a hem-o-lok clip to prevent loosening (B). For total inflow control, the catheter is pulled up alternatively using two dissecting forceps for several times and then clamped with another hem-o-lok clip close to the hepatoduodenal ligament to tighten it up (C). To relieve occlusion, the second hem-o-lok clip (close to the hepatoduodenal ligament) is transected by an ultrasound scalpel (D). 

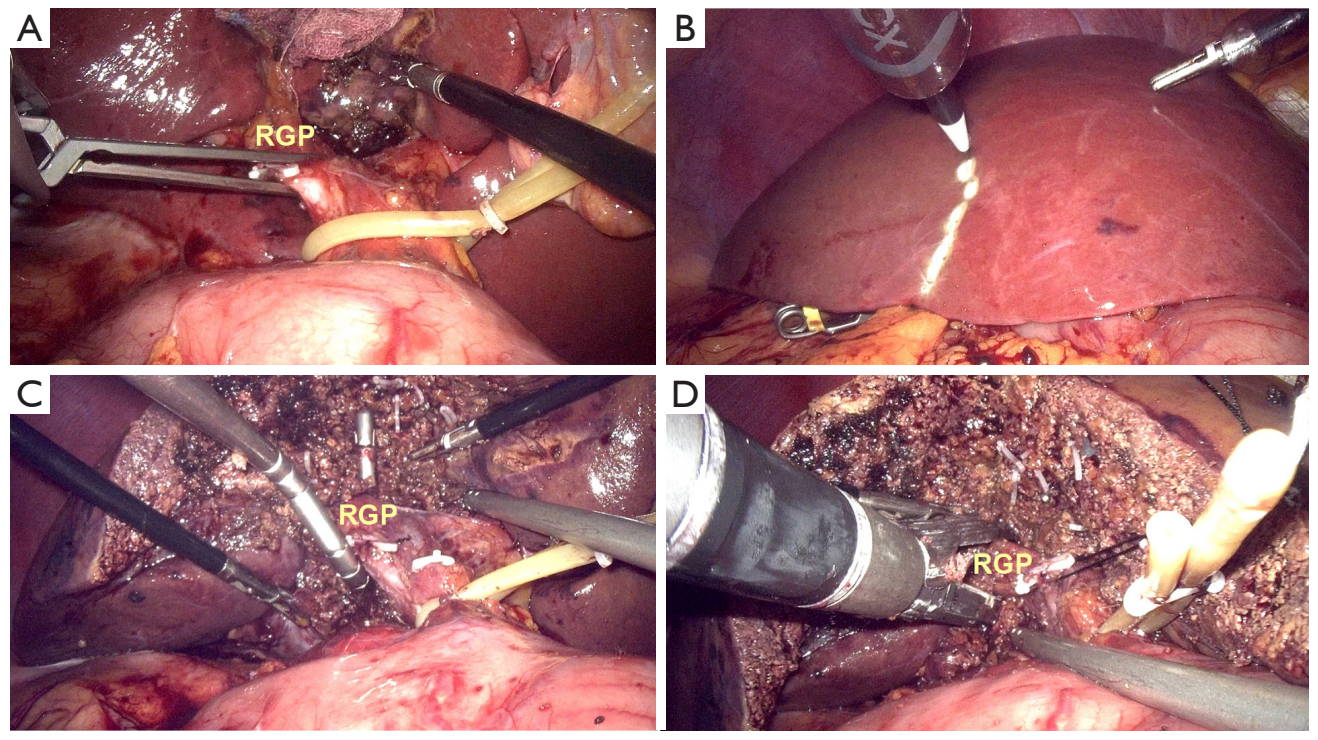

Figure 3 Liver parenchyma dissecting-first method for right hemihepatectomy. The right Glissonean pedicle (RGP) is clamped temporarily by a bulldog clamp (A) to identify the ischemic range of the right liver, and then the demarcation is marked using electrocautery (B). Parenchyma was then transected along the labeled ischemia line under intermittent total inflow control to reveal the middle hepatic vein, the parenchyma was dissected along its right side and toward the cephalic direction. After sufficient dissection, the RGP is encircled with a suture using a Goldfinger dissector for traction (C). The pedicle is then transected using a laparoscopic vascular stapler (D).

The removed specimen was put into a sterile canvas bag and taken out through a longitudinal subxiphoid incision (about $6-10 \mathrm{~cm}$ in length, Figure 1). One round $28-\mathrm{F}$ drainage tube was put beside the residual liver in all patients (10).

\section{Right bepatectomy}

For right hemihepatectomy (segments V to VIII), the right GP (RGP) was clamped temporarily by a bulldog clamp (Figure $3 A$ ) or a gastric grasping forceps to identify the ischemic range of the right liver (Figure 3B), Parenchyma was then transected along the labeled ischemia line under intermittent total inflow control to reveal the middle hepatic vein (MHV), the parenchyma was dissected along its right side and toward the cephalic direction. After sufficient dissection, the exposed RGP was easily encircled with a suture by using a Goldfinger dissector (Figure 3C). The RGP was then pulled upwards using the suture and transected using a laparoscopic vascular stapler (Ethicon Inc., Somerville, NJ, USA, Figure 3D). After that, parenchyma on the ventral side of IVC was transected. Hemostasis of the bleeding points on the MHV or IVC was achieved by applying titanium clip or bipolar electrocoagulator or suture, while short hepatic veins were clamped with titanium and hem-o-lok clips before transection. The right hepatic vein (RHV) was finally revealed and also transected using a vascular stapler.

For right anterior lobectomy (segments V and VIII), the RGP was clamped by a bulldog clamp or a gastric grasping forceps temporarily to identify the ischemic range of the right liver. Parenchyma was transected along the demarcation (the left resection line) under intermittent total inflow control to reveal the MHV, the parenchyma was dissected along its right side and toward the cephalic direction. After sufficient dissection, the intrahepatic right anterior GP (RAGP, Figure 4A) was easily encircled with a suture for traction. After that, the pedicle was transected using a vascular stapler (Figure 4B). Then the demarcation of right anterior and posterior lobe was revealed and marked (the right resection line) for further dissection.

For right posterior lobectomy (segments VI and VII), the parenchyma along the right side of RHV localized by using laparoscopic ultrasound was dissected toward the cephalic direction at first under intermittent total inflow control to fully reveal the right posterior GP (RPGP, Figure $4 C$ ). Then hem-o-lok clips were used to clamp the pedicle (Figure $4 D$ ) before transection.

For mesohepatectomy (segments IV, V, VIII), the 

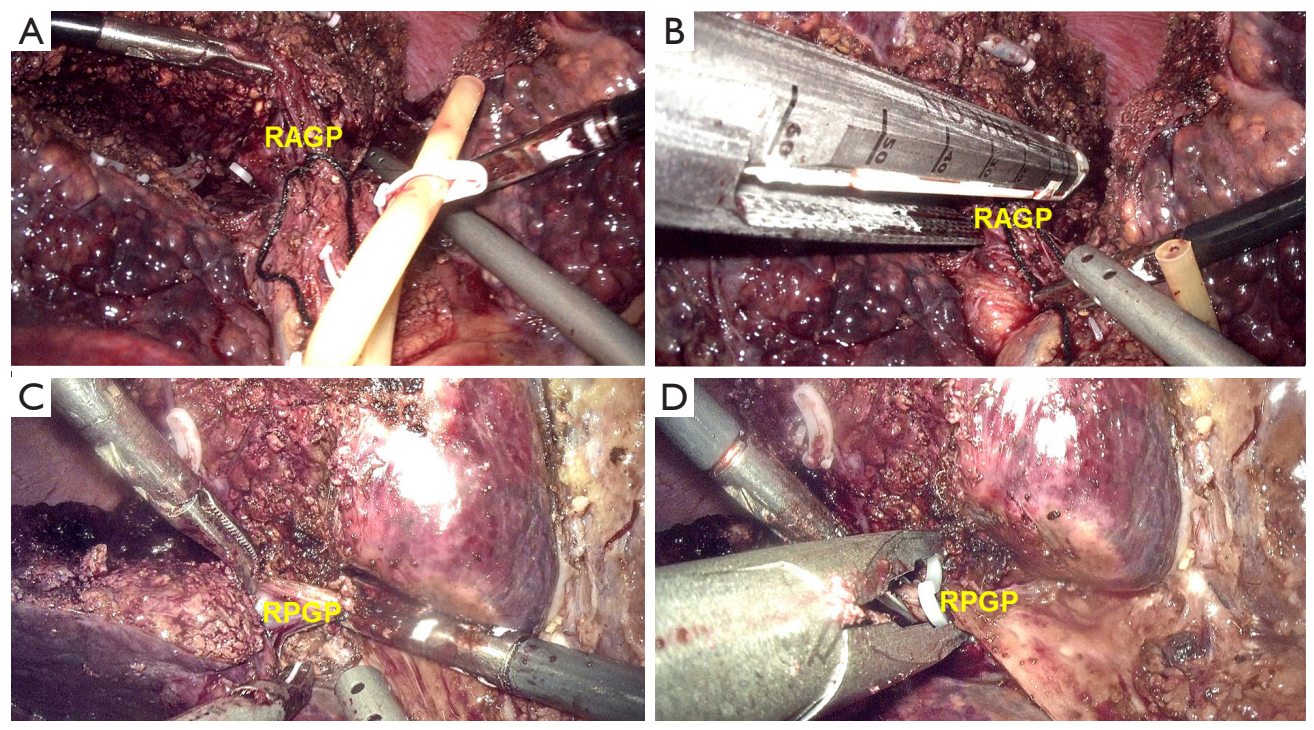

Figure 4 Liver parenchyma dissecting-first method for right hepatolobectomy. For right anterior lobectomy, the right Glissonean pedicle is clamped by a bulldog clamp temporarily to identify the ischemic range of the right liver, the parenchyma is transected along the labeled ischemia line (the left resection line) to reveal the right anterior Glissonean pedicle (RAGP), then the pedicle is encircled with a suture for traction (A). After that, the pedicle is transected using a vascular stapler (B) to reveal the demarcation of right anterior and posterior lobe (the right transection line) for further dissection. For right posterior lobectomy, the parenchyma along the right side of right hepatic vein localized by intraoperative ultrasound was dissected toward the cephalic direction at first to fully reveal the right posterior Glissonean pedicle (RPGP) (C). After sufficient dissection, the pedicle is clamped by hem-o-lok clips before transection (D).

parenchyma along the right side of the falciform ligament was first dissected toward the cephalic direction and the bifurcation of the left GP (LGP) and RGP (the left resection line). The IGPs to segment IV were clamped by hem-olok clips before transection. After sufficient dissection, the RAGP was encircled with a suture for traction, and then the pedicle was transected using a vascular stapler. Then the demarcation of the right anterior and posterior lobe was revealed and marked (the right resection line) for further dissection.

\section{Left bepatectomy}

For left hemihepatectomy (segments II to IV), the LGP was clamped by a bulldog clamp or a gastric grasping forceps temporarily to identify the ischemic range of the left lobe of the liver. Parenchyma was then transected along the labeled ischemia line to reveal the MHV, the parenchyma was dissected along its left side and toward the cephalic direction. After sufficient dissection, a laparoscopic vascular stapler was fired to transect the LGP. Then, parenchyma on the ventral side of IVC was transected. The left hepatic vein was finally revealed and also transected using a vascular stapler.

For left medial lobectomy (segment IV), the IGPs were revealed while dissecting the parenchyma along the right side of the falciform ligament (the left resection line) and then clamped using hem-o-lok clips. Then, the demarcation of the left and right lobe of the liver (the right resection line) was identified as described above for further dissection. Once the MHV was revealed, the parenchyma was dissected along its left side and toward the cephalic direction.

For left lateral lobectomy (segments II and III), the IGPs were revealed while dissecting the parenchyma along the left side of the falciform ligament and then clamped with hem-o-lok clips or a laparoscopic vascular stapler before transection. Usually, intermittent total inflow control was not necessary.

\section{Results}

LPDF was used to facilitate GPA in all patients. Seven patients underwent laparoscopic right hemihepatectomy, three underwent laparoscopic left hemihepatectomy, three underwent laparoscopic mesohepatectomy (segments IV, V and VIII), and two underwent laparoscopic right 
posterior lobectomy. The mean operation time was 245 minutes (ranging from 230 to 350 minutes). Vital signs were stable, and no blood transfusion was needed during the surgery. Antibiotics, albumin and liver protection drugs (ademetionine and magnesium isoglycolate) were given after the operation, while entecavir was administrated daily to 12 patients with CHB. The drainage tube was removed within 4-5 days post-operation. The median time of hospital stay was 7 days (ranging from 5 to 8 days). The pathological results were CHB-related HCC in 12 patients, metastatic colon carcinoma to the liver in 1 patient, and giant hemangioma in 2 patients. The surgical margins of the 13 patients with malignant lesions were all negative and exceeded $1 \mathrm{~cm}$. No patient died, and no postoperative intraperitoneal bleeding or biliary leakage occurred in this study. Patients were followed up for 3 to 13 months. The 3 patients underwent laparoscopic mesohepatectomy had effusion in operation area found by color doppler ultrasound one month after the operation, but no fever or abdominal pain was noted, and the number of white blood cells and level of total serum bilirubin were within the normal ranges. The effusion was absorbed gradually without treatment.

\section{Discussion}

Isolation of the GP before parenchyma dissection is a crucial step in anatomical liver surgery. Takasaki et al. $(5,6)$ first described using this technique during open liver surgery in 1990, reporting safe parenchyma transection with less intraoperative bleeding. Subsequently, it was widely accepted as the "Glissonean pedicle approach (GPA)". Although the first laparoscopic hepatectomy (LH) was reported in 1991 (11), the popularization of GPA in LH was slow, because it was considered a difficult procedure at that time due to the limited motion and vision of the laparoscopic instruments and the possibility of false injury to the GP (12).

In 2006, Yoon et al. (13) first reported their experience in laparoscopic isolation of the GP before parenchymal transection and emphasized the importance of the GPA in ALH. One year later, Cho et al. (14) introduced dissection and encircling of the main branch of GP in laparoscopic hemihepatectomy, naming this method "extrahepatic GPA" $(15,16)$. However, this method is complicated and timeconsuming, and may result in false injury to the vascular and biliary structures when anatomical variation is present and the surgeons are lack of experience.

Following these developments, Machado et al. $(17,18)$ modified the GPA by making incisions beside the pedicle to facilitate clamping. They named it "intrahepatic GPA" (IGPA), and applied this method in laparoscopic right hepatectomy in 2008 (7). This method is attractive because it is able to overcome the difficulty in isolating and completely encircling the pedicle. However, it is likely to injure the underlying small GPs and hepatic veins and may lead to parenchyma injury and bleeding (12). Taking this into consideration, we speculated that it may be valuable to expose these vascular structures before surgery using a three-dimensional surgery planning software like XPLiver (Myrian, France). In this way, the surgeons can be made aware of whether there are anatomical variations, and dissect the IGP precisely with more confidence.

Another reasonable solution to the shortcomings of above methods is to reveal the GP adequately before transection, which requires dissecting the liver parenchyma at first. Theoretically, this method can avoid unnecessary damage to the GP and reduce intraoperative hemorrhage. For example, during left hemihepatectomy, it is very important to fully expose the GP before applying the stapler in case the RAGP or RPGP converges with the LGP (8). Moreover, there are also many anatomic variations of the RGP, so we applied LPDF in recent clinical practice. The innovation of LPDF that distinguishes it from the traditional GPA $(19,20)$ is that the secondary GPs (LGP, RGP, RAGP and RPGP) are only encircled and transected after sufficient parenchyma dissection along the relatively fixed resection line in ALH, which reduces the difficulty of extrahepatic encircling of the secondary GPs and the possibility of false injury to the structures in them. Indeed, no false injury to the GP occurred in our study, and no intraoperative blood transfusion was needed. LPDF was safe and easy to apply under intermittent total inflow control, while the isolation and transection of GP became much easier after sufficient parenchyma dissection. Besides, it is worth mentioning that the approach to the tertiary branches of the secondary GPs described by Yamamoto et al. (19) was also parenchyma dissecting first.

In addition, before the parenchymal dissection, we used an improved method for laparoscopic Pringle maneuver (Figure 2). It is simpler to implement when compared with the "extracorporeal tourniquet method" which requires an extra incision on the abdominal wall (21). The only disadvantage of this method may be that it needs several more hem-o-lok clips. According to our experience, the bleeding during parenchymal dissection can be easily controlled by electrocautery or bipolar electrocoagulator 
under total inflow control, and this has minimal effects on the post-operative recovery. Therefore, it is not necessary to be concerned with the number of occlusion occurrences. After sufficient parenchyma dissection, the whole target GP can be revealed for encirclement with little risk of false injury to the posterior side of the vessels. Then, the stapler can be fired more safely. This was confirmed by Lee et al. (8), who applied temporary inflow control of the GP for totally laparoscopic hemihepatectomy by GPA. When compared to their study (8), we herein provided not only a modified method for laparoscopic Pringle maneuver, but also more information about how to reveal the RAGP, RPGP, and LGP, and these methods were used in combination for laparoscopic mesohepatectomy. We suppose the main merit of LPDF compared with other methods is the convenience of acquiring direct and accurate paths to the GP, thus facilitating ALH. For a better understanding of LPDF, the line graphs (Figures S1-S3) for Figures 2-4 and a video (Video 1) for laparoscopic right hemihepatectomy are provided as Supplementary file.

However, there are undeniable limitations of LPDF. For example, the intrahepatic metastasis of HCC, which spreads along the portal vein, might occur during parenchyma dissection under total inflow occlusion when the portal vein tumor thrombus exists. In that case, traditional GPA is recommended for the target GP is ligated before parenchyma dissection, which is more conform to the principle of surgical oncology. In addition, the borderline of the right anterior and posterior lobe (the RHV) is relatively hard to localize and it was identified by using laparoscopic ultrasound in our study, which leads to higher requirements for surgical instruments, especially in grass-roots hospitals.

\section{Conclusions}

We have achieved satisfactory results in the current study. Considering its safety and simplicity, we believe LPDF (together with the modified laparoscopic Pringle maneuver) can facilitate GPA, and enhance the confidence of surgeons with less experience to perform ALH with less operative time and intraoperative bleeding, thus promoting the application of ALH. Further clinical control trails are needed to validate the superiority of LPDF compared with GPA in ALH.

\section{Acknowledgments}

Funding: This work was supported by the Nature Science
Foundation of Hunan Province (No. 2018JJ3812). The funder had no role in study design, data collection and analysis, decision to publish, or preparation of the manuscript.

\section{Footnote}

Reporting Checklist: The authors have completed the STROBE reporting checklist. Available at http://dx.doi. org/10.21037/atm-20-4674

Data Sharing Statement: Available at http://dx.doi. org/10.21037/atm-20-4674

Conflicts of Interest: All authors have completed the ICMJE uniform disclosure form (available at http://dx.doi. org/10.21037/atm-20-4674). The authors have no conflicts of interest to declare.

Ethical Statement: The authors are accountable for all aspects of the work in ensuring that questions related to the accuracy or integrity of any part of the work are appropriately investigated and resolved. These patients provided informed consent for the indication of the surgery, the possible postoperative complications, prognosis, and the timing of conversion to open surgery, and for the publication of their cases and any accompanying images. All procedures performed in this study involving human participants were in accordance with the Declaration of Helsinki (as revised in 2013). The study protocol was approved by the hospital institutional review board (approval ID: 202002023).

Open Access Statement: This is an Open Access article distributed in accordance with the Creative Commons Attribution-NonCommercial-NoDerivs 4.0 International License (CC BY-NC-ND 4.0), which permits the noncommercial replication and distribution of the article with the strict proviso that no changes or edits are made and the original work is properly cited (including links to both the formal publication through the relevant DOI and the license). See: https://creativecommons.org/licenses/by-nc-nd/4.0/.

\section{References}

1. Fu SY, Lau WY, Li GG, et al A prospective randomized controlled trial to compare Pringle maneuver, hemihepatic vascular inflow occlusion, and main portal vein inflow 
occlusion in partial hepatectomy. Am J Surg 2011;201:62-9.

2. Simillis C, Li T, Vaughan J, et al. A Cochrane systematic review and network meta-analysis comparing treatment strategies aiming to decrease blood loss during liver resection. Int J Surg 2015;23:128-36.

3. Kotewall CN, Cheung TT. Optimizing hepatectomy for hepatocellular carcinoma in Asia-patient selection and special considerations. Transl Gastroenterol Hepatol 2018;3:75.

4. Zhan C, Dai X, Shen G, et al. Preoperative shortterm fasting protects liver injury in patients undergoing hepatectomy. Ann Transl Med 2018;6:449.

5. Takasaki K. Glissonean pedicle transection method for hepatic resection: a new concept of liver segmentation. J Hepatobiliary Pancreat Surg 1998;5:286-91.

6. Takasaki K, Kobayashi S, Tanaka S, et al. Highly anatomically systematized hepatic resection with Glissonean sheath code transection at the hepatic hilus. Int Surg 1990;75:73-7.

7. Machado MA, Makdissi FF, Galvao FH, et al. Intrahepatic Glissonian approach for laparoscopic right segmental liver resections. Am J Surg 2008;196:e38-42.

8. Lee N, Cho CW, Kim JM, et al. Application of temporary inflow control of the Glissonean pedicle method provides a safe and easy technique for totally laparoscopic hemihepatectomy by Glissonean approach. Ann Surg Treat Res 2017;92:383-6.

9. Chen H, Merchant NB, Didolkar MS. Hepatic resection using intermittent vascular inflow occlusion and low central venous pressure anesthesia improves morbidity and mortality. J Gastrointest Surg 2000;4:162-7.

10. Cho A, Yamamoto H, Kainuma O, et al. Arantius' ligament approach for the left extrahepatic Glissonean pedicle in pure laparoscopic left hemihepatectomy. Asian J Endosc Surg 2012; 5:187-90.

11. Reich H, McGlynn F, DeCaprio J, et al. Laparoscopic

Cite this article as: Xiao L, Wang Z, Zhou L. "Liver parenchyma dissecting-first" method facilitates the Glissonean pedicle approach in anatomical laparoscopic hepatolobectomy. Ann Transl Med 2020;8(15):940. doi: 10.21037/atm-20-4674 excision of benign liver lesions. Obstet Gynecol 1991;78:956-8.

12. Choi Y, Han HS, Sultan AM, et al. Glissonean pedicle approach in laparoscopic anatomical liver resection. Hepatogastroenterology 2014;61:2317-20.

13. Yoon YS, Han HS, Choi YS, et al. Total laparoscopic right posterior sectionectomy for hepatocellular carcinoma. J Laparoendosc Adv Surg Tech A 2006;16:274-7.

14. Cho A, Asano T, Yamamoto H, et al. Laparoscopyassisted hepatic lobectomy using hilar Glissonean pedicle transection. Surg Endosc 2007;21:1466-8.

15. Cho A, Yamamoto H, Kainuma O, et al. Safe and feasible extrahepatic Glissonean access in laparoscopic anatomical liver resection. Surg Endosc 2011;25:1333-6.

16. Cho A, Yamamoto H, Kainuma O, et al. Extrahepatic Glissonean approach for laparoscopic major liver resection (with video). J Hepatobiliary Pancreat Sci 2013;20:141-4.

17. Machado MA, Herman P, Machado MC. Intrahepatic Glissonian approach for pedicle control during anatomic mesohepatectomy. Surgery 2007;141:533-7.

18. Machado MA, Herman P, Makdissi FF, et al. Intrahepatic Glissonian approach for anatomical resection of left liver segments. Langenbecks Arch Surg 2008;393:1017; author reply 1019-20.

19. Yamamoto M, Katagiri S, Ariizumi S, et al. Tips for anatomical hepatectomy for hepatocellular carcinoma by the Glissonean pedicle approach (with videos). J Hepatobiliary Pancreat Sci 2014;21:E53-6.

20. Kim JH, Kim H. Pure Laparoscopic Anatomical Segment V Resection Using the Extrafascial and Transfissural Glissonean Approach. Ann Surg Oncol 2019;26:2241.

21. Rotellar F, Pardo F, Bueno A, et al. Extracorporeal tourniquet method for intermittent hepatic pedicle clamping during laparoscopic liver surgery: an easy, cheap, and effective technique. Langenbecks Arch Surg 2012;397:481-5. 

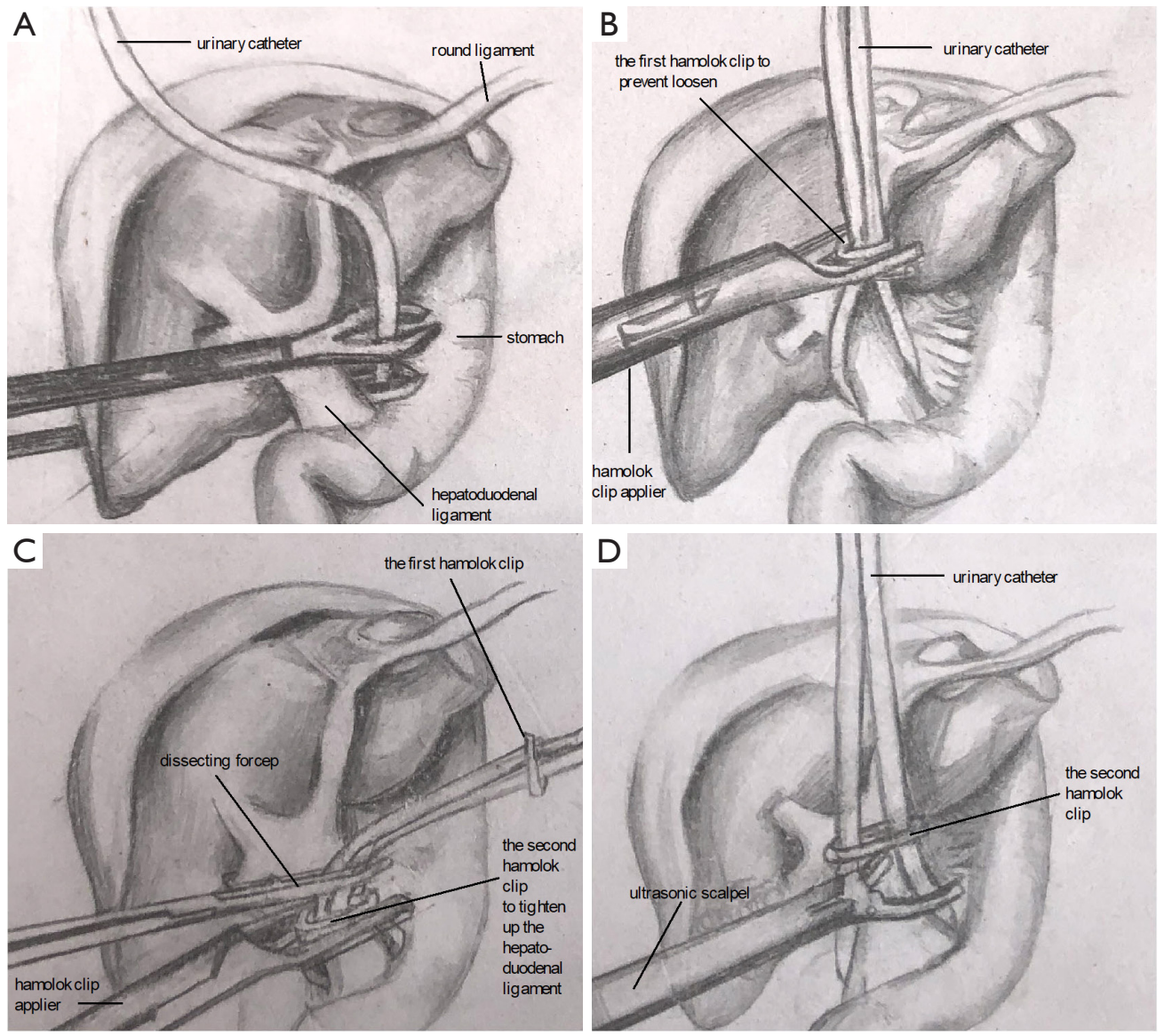

Figure S1 This is a line graph for Figure 2 (a modified method for laparoscopic Pringle maneuver). The hepatoduodenal ligament is encircled with a urinary catheter (approximately $10 \mathrm{~cm}$ in length, A). The catheter is clamped with a hem-o-lok clip to prevent loosening (B). For total inflow control, the catheter is pulled up alternatively using two dissecting forceps for several times and then clamped with another hem-o-lok clip close to the hepatoduodenal ligament to tighten it up (C). The second hem-o-lok clip is transected by an ultrasound scalpel to relieve occlusion (D). 

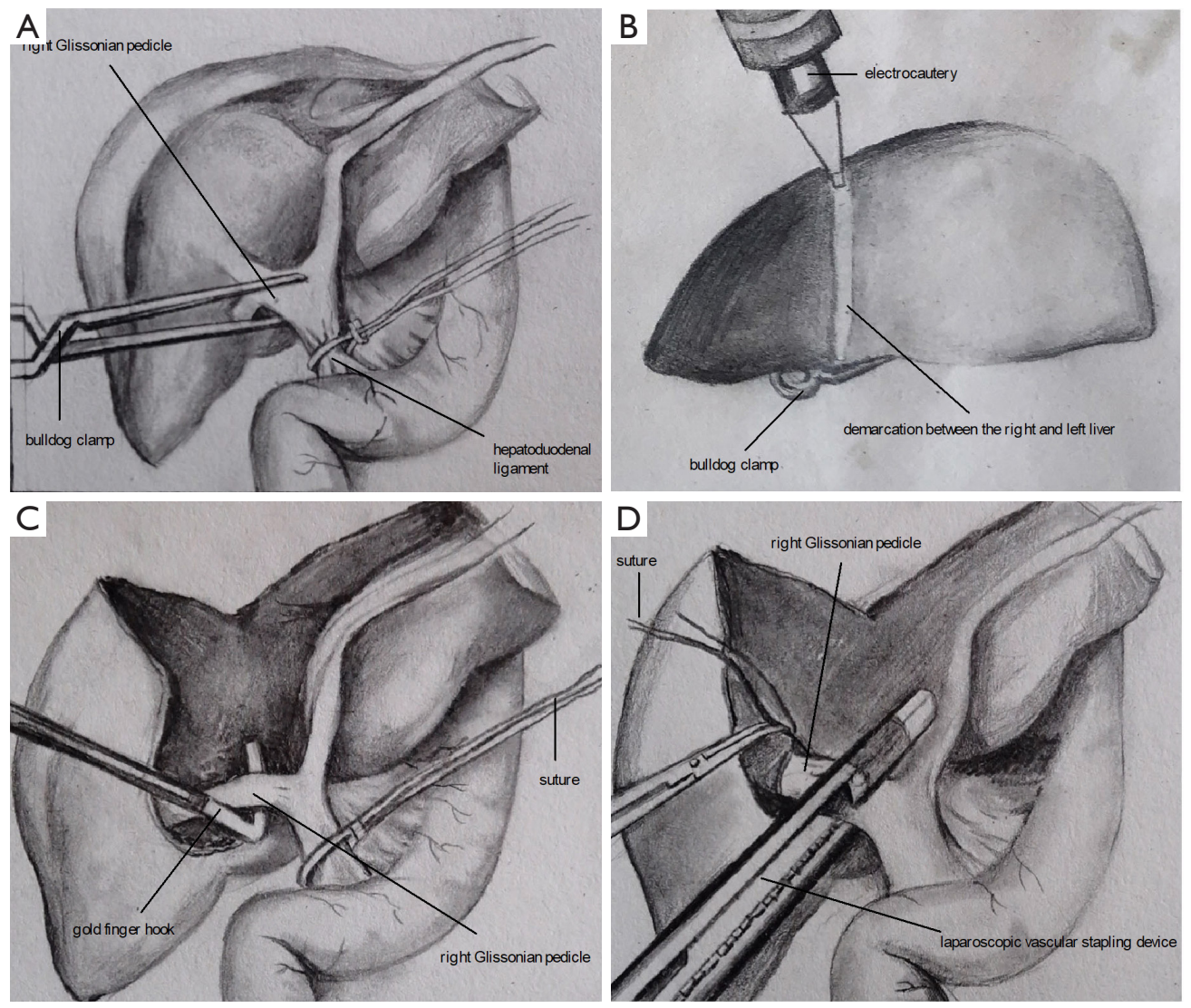

Figure S2 This is a line graph for Figure 3 (liver parenchyma dissecting-first method for right hemihepatectomy). The right Glissonean pedicle (RGP) is clamped temporarily by a bulldog clamp (A) to identify the ischemic range of the right liver, and then the demarcation is marked using electrocautery (B). Parenchyma was then transected along the labeled ischemia line under intermittent total inflow control. After sufficient dissection, the RGP is encircled with a suture using a Goldfinger dissector for traction (C). The pedicle is then transected using a laparoscopic vascular stapler (D). 

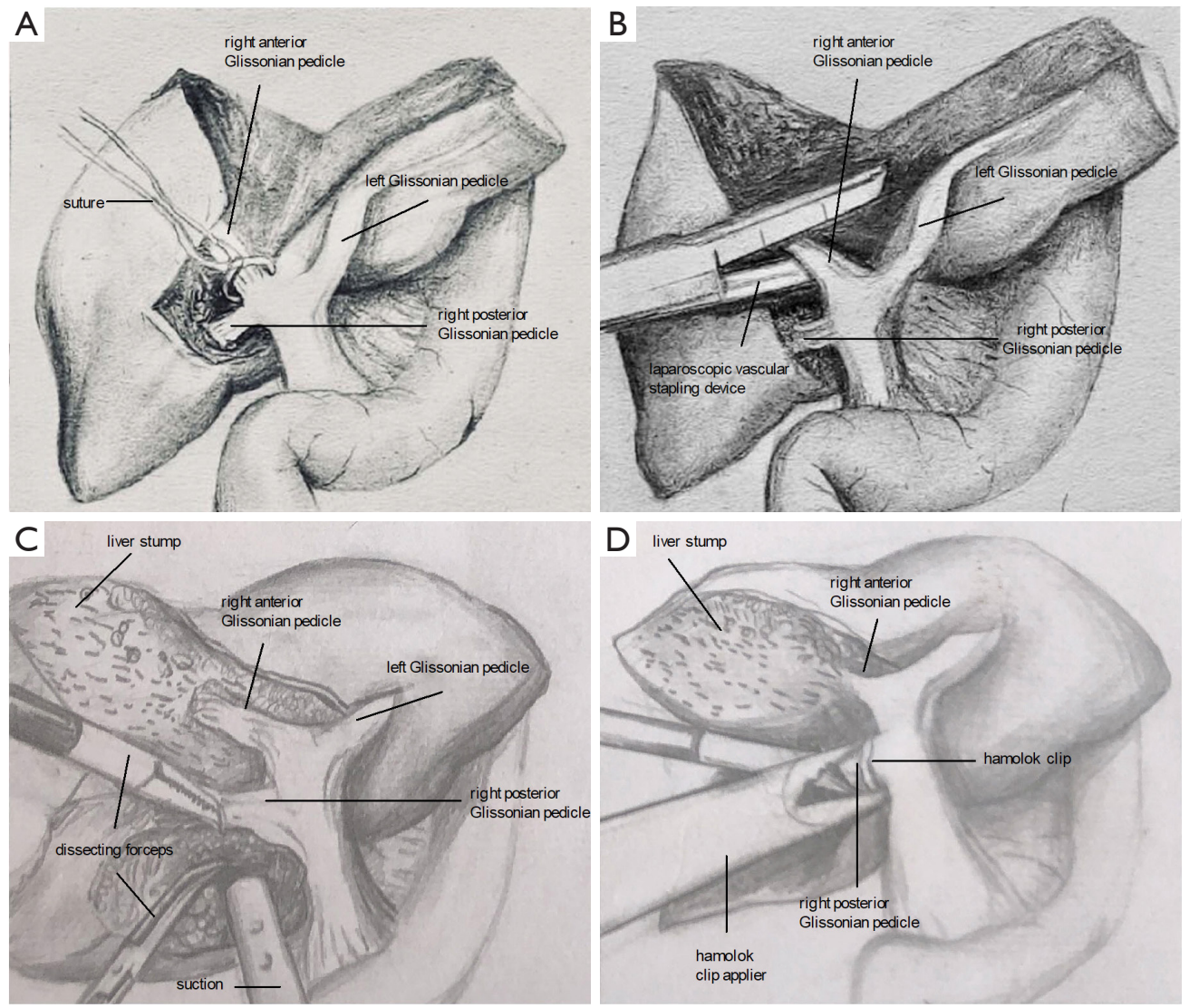

Figure S3 This is a line graph for Figure 4 (liver parenchyma dissecting-first method for right hepatolobectomy). For right anterior lobectomy, the parenchyma is transected along the right side of middle hepatic vein to reveal the right anterior Glissonean pedicle, then the pedicle is encircled with a suture for traction (A). After that, the pedicle is transected using a vascular stapler (B) to reveal the demarcation of right anterior and posterior lobe for further dissection. For right posterior lobectomy, the parenchyma along the right side of right hepatic vein was dissected toward the cephalic direction at first to fully reveal the right posterior Glissonean pedicle (C). After sufficient dissection, the pedicle is clamped by hem-o-lok clips before transection (D). 